\title{
Cost of dynamical quark simulations with improved staggered quarks
}

\author{
Steven Gottlieb $b^{\mathrm{a}}$ * \\ ${ }^{a}$ Department of Physics-SW117; Indiana University; Bloomington, IN 47405; USA

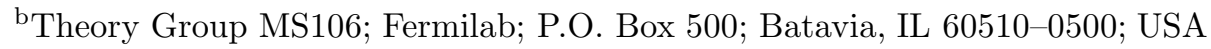

The cost of dynamical quark simulations with improved staggered quarks is estimated based on current and planned running by the MILC collaboration. I find that a few 10 s of Teraflop years should be sufficient to calculate down to a lattice spacing of $0.045 \mathrm{fm}$.

\section{INTRODUCTION}

Several caveats should be pointed out to the reader. First, I think that the past 20 years of my life are evidence of my inability to estimate the time and effort necessary to calculate the spectrum of QCD. Used to doing analytic calculations that took a few months at most, I did not imagine as I started my first Monte Carlo calculation that 20 years later I would still be doing similar calculations. I was a postdoc at Fermilab then, and as I write this I am back at Fermilab on sabbatical. With great pleasure I see how far we have come, and look forward to an exciting future of improved calculations.

Caveat two is that this write-up is not a transcript of what I said in Berlin. Caveat three is that this does not represent a MILC consensus statement. I did my best to extract from past experience what is required for future calculations, but the whole collaboration has not had an opportunity to check or react.

\section{TIME ESTIMATE}

Since the CG routine is no longer so dominant, the formula for counting operations is not quite so simple. For a $2+1$ flavor run, let: $\tau=\#$ of time units per independent configuration; $O_{F}=\#$ of operations for fermion force per site; $O_{F L}=\#$ of operations for fat link calculation per site; $O_{C G}=$ \# of operations per CG iteration per site; $N_{C G}^{s}=$

\footnotetext{
*At Fermilab until June 15, 2002.
}

\# of iteration to solve CG for $s$-quark. Denoting the quark masses $m_{s}$ and $m_{l}$, we use a time step $\Delta t=2 / 3 m_{l}$, and we expect $N_{C G}^{l}=N_{C G}^{s} \cdot m_{s} / m_{l}$. The operation count is

$$
\begin{aligned}
=\frac{3}{2} \frac{L}{a}^{4} \tau \frac{1}{m_{l}} & {\left[\left(2 O_{F}+O_{F L}+O_{C G} N_{C G}^{s}\right)\right.} \\
& \left.+O_{C G} N_{C G}^{s} \frac{m_{s}}{m_{l}}\right]
\end{aligned}
$$

\subsection{Production running}

MILC, already in production with three dynamical quarks, has completed runs with lattice spacing 0.2 and $0.13 \mathrm{fm}$ [1]. The Asqtad action has leading errors of order $a^{2} g^{2}$ and uses tadpole improved coefficients in the action [2]. A series of runs was done to allow a smooth interpolation between the quenched approximation and the $2+1$ flavor world. The coupling is tuned as the quark masses are reduced to fix a length scale determined from the heavy quark potential [3]. We have eight dynamical runs. In four, there are three degenerate quarks with mass $8 m_{s}, 4 m_{s}$, $2 m_{s}$ or $m_{s}$. In four runs, the dynamical strange quark is fixed at $m_{s}$ and the light quark mass is $0.8,0.6,0.4$ or 0.2 times $m_{s}$.

Runs are $\approx 2000-3000$ molecular dynamical time units. One significant issue is how the autocorrelation time scales as the lattice spacing is reduced. We cannot yet provide numerical evidence for this scaling law. Currently, we are saving every sixth trajectory. We see some autocorrelation between successive lattices. For analysis, we bin in groups of four, i.e., 24 time units.

Also important is how many independent configurations are needed to achieve the desired accu- 
racy. This number clearly depends on the quark masses and the quantity under study, but we (the lattice community) may not be aware of its dependence on such quantities as the action and volume. As an example of the latter, MILC has done some extensive tests of finite size effects in the past. It is much easier to get accurate masses on large volumes than on the smaller ones. Thus, we need more configurations for the smaller volumes. In our projects, we often use big volumes compared to some groups using Wilson or Clover quarks. We may need fewer independent configurations to achieve the same accuracy. (Not to mention no finite-size effects.)

Table 1 has timing estimates for some current runs at $0.09 \mathrm{fm}$. We print timing for the conjugate gradient routine, but not for other parts of the code. We run on several different machines, but this estimate is based on the assumption of a speed of $200 \mathrm{MF} / \mathrm{CPU}$. To get the operation count we will assume the entire code is running at $200 \mathrm{MF}$, not just the CG (the only part regularly timed). The table contains the number of node hours required to create a configuration we will store given the parameters we use for time step and residual. The lattice volume is $28^{3} \times 96$. In the first line, the quarks are degenerate, so we need only one quark field and $N_{f}=3$. (For Asqtad, $O_{F} \approx 420 \mathrm{~K}, O_{F L} \approx 51 \mathrm{~K}, N_{C G}=1187$ and $N_{C G}^{s}=236$, giving an opcount within $30 \%$ of the table value.)

For the other two runs, we need two quark fields, with $N_{f}=2$ and 1 . In the last run, we reduce the light quark mass by a factor of two compared to the one above it. Traditional scaling laws would predict a factor of 4 increase in computation from halving the time step and doubling the number of CG iterations; however, as the code is no longer dominated by the CG routine, the time only increases by 2.4. For the lightest mass run, we plan to store 400 configurations. This amounts to 0.145 TF-years.

Now we attempt to address the issue of what it would take to do a calculation of the quality of the CPPACS quenched Wilson quark calculation. CPPACS states that their smallest lattice spacing is $0.05 \mathrm{fm}$. However, they only have 150 configurations there, and their error bars are sig-
Table 1

Computational requirement of runs

\begin{tabular}{ccc}
\hline$m_{u, d}$ & node-hr $/$ conf & flops $\left(10^{14} /\right.$ conf $)$ \\
\hline$m_{s}$ & 900 & 6.5 \\
$0.4 m_{s}$ & 4096 & 29.5 \\
$0.2 m_{s}$ & 9780 & 70.4
\end{tabular}

nificantly larger than at the stronger couplings. I have always wondered how their continuum extrapolation would change eliminating either the smallest or largest $a$. Is the hardest part of the calculation important in reducing the final error? If we halve our current lattice spacing to get to $0.045 \mathrm{fm}$, we will be somewhat closer to the continuum limit than they were. Assuming we generate 400 configurations, we can roughly estimate the time required by multiplying our current light mass run by a factor of $2^{6}$ to $2^{8}$. The smaller factor assumes four powers from the volume, one from the time step and another from the CG iterations (which may be an overestimate as the CG no longer dominates). The larger factor allows for a doubling of the autocorrelation time, and doubles the time for additional runs at heavier masses. This yields an estimate of 10-40 TF-yr of running. CPPACS's next to smallest $a$ was $0.064 \mathrm{fm}$. If we were to go to a lattice spacing of $0.06 \mathrm{fm}$, the increase in time from our present run would be from 11 to 26 depending on how things scale. This would only require about $2-4 \mathrm{TF}-\mathrm{yr}$.

Despite my first caveat and the surprise of many in the audience, I believe these are reasonable estimates for the runs outlined. MILC has not had a dedicated computer, but we have been able to do significant calculations partly because staggered quarks do not require as much computation as Wilson/clover.

\section{REFERENCES}

1. C. Bernard et al., Phys. Rev. D 64 (2001) 054506, hep-lat/0104002.

2. K. Orginos, D. Toussaint and R.L. Sugar, Phys. Rev. D 60 (1999) 054503, heplat/9903032; G.P. Lepage, ibid, 59 (1999) 074502, hep-lat/9809157. 
3. C. Bernard et al., Phys. Rev. D 62 (2000) 034503, hep-lat/0002028. 